\title{
Design of a Low Cost Instrumentation System for Oxygen Sensing Using Polyaniline/Cerium Oxide Composites
}

\author{
Khened B.S ${ }^{1}$, Nagabhushana Katte ${ }^{2}$, M V N Pradeep ${ }^{3}$, M V N Ambika Prasad ${ }^{4}$, \\ and M Sasikala ${ }^{5}$ \\ ${ }^{I}$ Department of Electrical and Electronics Engineering, BITM, Bellary-583 104, KA, India \\ ${ }^{2}$ Department of Electronics and Communication Engineering, BITM, Bellary-583 104, KA, India \\ ${ }^{3}$ Department of Instrumentation and Control, M I T,Manipal-576104,KA,India \\ ${ }^{4}$ Department of Materials science, Gulbarga University, Gulbarga-585 105, KA, India \\ ${ }^{5}$ Netaji Institute Of Engineering and Technology, Toopranpet(Vill), 508252 Nalgonda (Dist). AP,INDIA
}

\begin{abstract}
In the present paper design and development of a low cost gas sensing system with Polyaniline/ Cerium oxide composite as sensing element is presented. Polyaniline / Cerium oxide composites are prepared by insitu polymerization. The composites are pressed in the form of pellets and are used as the sensing elements. The sensing element is interfaced with front end signal conditioning circuit, and ARM processor for data logging. The sensor readout circuit is based on resistance to voltage conversion technique. The performance of the system was tested for oxygen sensing application.
\end{abstract}

Keywords: Polyanilinel Cerium oxide composite, oxygen sensing, signal conditioning, ARM processor, resistance measurement.

\section{Introduction}

Need for monitoring and maintaining the pollutant gases in the environment within a specified limit has led to substantial research in the field of gas sensors. Several studies have been reported in the literature for detection of various gases using metal oxide sensors [1-11]. Huge number of gas sensors are commercially available in the market which employ metal oxides.However,these sensors operate at high temperatures and their electrical conductivity depends on the concentration of ambient gas. Since conducting polymer sensors have short response time, high sensitivity and are operated at room temperature, researchers have paid much attention on these novel materials for gas sensing applications. Polyaniline has gained importance in gas sensing applications due to its environmental stability, good conductivity and gas sensing ability. Gas sensing properties of conducting polyaniline composites and polypyrrole composites for various oxidizing and reducing gases have been extensively studied and detailed mechanism has been reported [12-20]. When the conducting polymer composites are exposed to the oxidizing or reducing gases, the conductivity of these sensing elements will change. The overall conduction in a sensor element is determined by various factors such as nature of dopant in polyaniline, surface reactions, resulting charge transfer processes with the underlying semi conducting material, transport mechanism through the sensing material, and morphology of sensing layer [21]. Since these sensors are operated at room temperature, the sensor resistance $\left(\mathrm{R}_{\mathrm{SENS}}\right)$ is given by the following equation,

$$
\mathrm{R}_{\mathrm{SENS}}=\mathrm{R}_{\mathrm{AIR}}+\Delta \mathrm{R}_{\mathrm{GAS}}
$$

Where $\mathrm{R}_{\mathrm{AIR}}$ is the sensor resistance in air at room temperature and $\Delta \mathrm{R}_{\mathrm{GAS}}$ is the variation in sensor resistance due to interaction between sensor element and the gas. The value of $\mathrm{R}_{\text {AIR }}$ depends on method of preparation of sensing element, dopant and its concentration. The value of $\Delta \mathrm{R}_{\mathrm{GAS}}$ depends on the gas concentration. In the present paper, the development of a low cost sensor system with Polyaniline/ Cerium oxide composite as sensing element and its interfacing with front end signal conditioning circuit is presented. The system is tested for oxygen sensing application.

\section{Sample Preparation}

Polyaniline/Cerium oxide composites were prepared by insitu polymerization. Aniline solution was formed by dissolving aniline $(0.1 \mathrm{~mol})$ in $1 \mathrm{M} \mathrm{HCl}$. Cerium oxide was added to the aniline solution with vigorous stirring to keep Cerium oxide suspended in the solution. 0.1M Ammonium persulfate, which acted as the oxidant, was added to this reaction mixture slowly with continuous stirring at $0-5^{\circ} \mathrm{C}$. The reaction mixture was kept stirring for 24 hours. The polymer which was in the form of greenish-black precipitate was recovered by vacuum filtration and washed with deionized water. To achieve a constant weight the precipitate was dried for 24 hours in an oven. In this way polyaniline - Cerium oxide composites with 5 different weight $\%$ of $\mathrm{CeO}_{2}(10$, $20,30,40,50)$ in polyaniline were synthesized. The composites thus formed were characterized by Fourier Transform Infra Red Spectroscopy (FTIR), X-ray diffraction (XRD) and scanning electron microscopy (SEM), 
which confirmed the presence of $\mathrm{CeO}_{2}$ in polyaniline matrix. The composites are pressed in the form of pellets. These pellets are used as the sensing elements.

\section{A. Principle}

\section{Measurement Instrumentation}

The gas measurement instrumentation consists of constant current source, signal conditioner, data acquisition unit, ARM processor and a display unit. Block diagram of the gas sensing and measurement system is shown in figure 1. Conducting polymer composite pellet is used as gas sensing element and is placed in the gas sensing chamber. Gas sensor is driven by a current of fixed magnitude supplied by the constant current source.

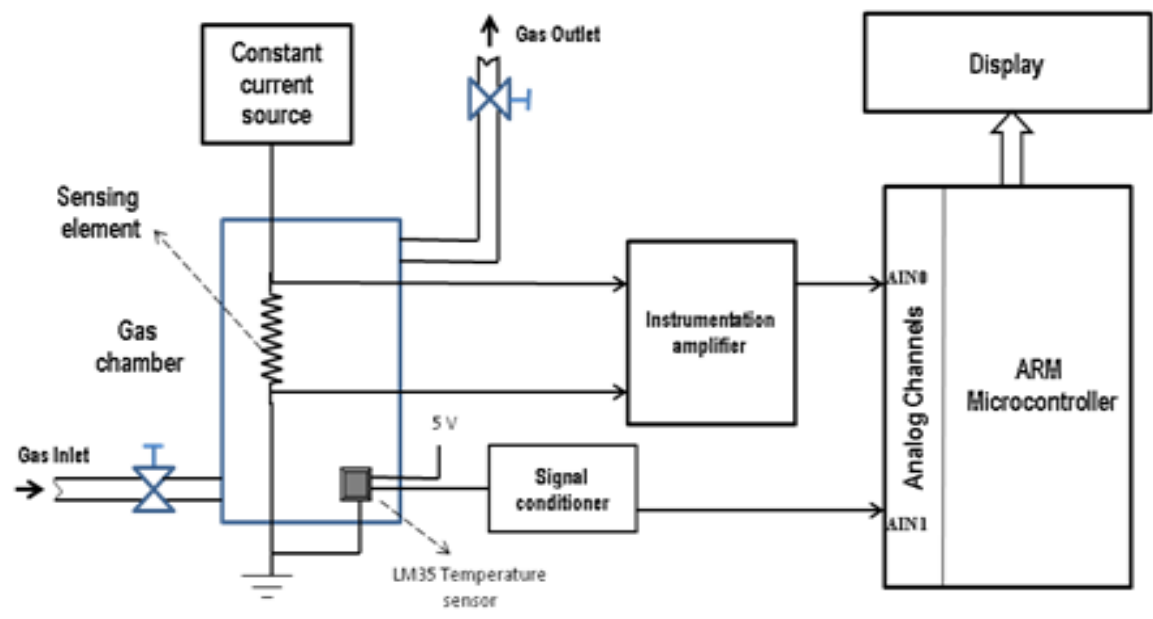

Figure 1. Block diagram of gas sensing system

With variation in gas concentration, the resistance of the pellet and hence the voltage across it also varies. The change in voltage is picked up by the signal conditioner where the signal is suitably modified and is further acquired by the data acquisition unit available on the ARM processor. Acquired data is processed by the processor and the same is displayed in parts per million (PPM).

\section{A. Circuit design}

Metal oxide gas sensors operate at high temperature. The baseline value of resistance in these sensors varies from kilo ohms to several mega ohms. The resistance value of sensors depends on temperature, type of material used for sensing, nature of gas to be sensed and its concentration. Several techniques have been proposed for measurement of large dynamic variations in resistance. Shurmer and Gardner have used potential divider method for interfacing of chemoresisters [22]. It was simple to implement, but not very sensitive for small variations in resistance. Wheatsone bridge technique was proposed by Gardner et.al. [23], but with large resistance swing, output suffers from non linearity. Diego Barrettino et.al. proposed logarithmic converter circuit, which uses voltage to current conversion technique and was used for measuring change in resistance from 1 kilo ohm to 10 mega ohms[24]. However, the precision is reduced due to the usage of logarithmic function. As metal oxide sensors operate at high temperature, heater design must also be made for accurate control of temperature. Since the proposed system is operated at room temperature, heater design is eliminated.

Signal conditioner circuit (resistance to voltage conversion and instrumentation amplifier) designed for gas measurement is shown in figure 2. . A design with the combination of transistor (Q2N2222) and Zener diode $(1 \mathrm{~N} 4733 \mathrm{~A})$ is used as a constant current source. Gas sensing element is used as a feedback element in the design of the constant current source. Resistance at the emitter terminal of the transistor is set to drive the sensing element with a constant current of $10 \mu \mathrm{A}$. Gas sensing element is used as a feedback element in the design of the constant current source.. voltage drop across the sensing element is directly proportional to the sensor resistance and is picked up by the instrumentation amplifier. The instrumentation amplifier is designed using 3 operational amplifiers (OP-07). The gain of the amplifiercan be set to the desired value by adjusting the $100 \mathrm{~K}$ resistor. The output of the amplifier is applied to the ARM processor. Further ARM processor acquires the data, processes, and displays the gas concentration in PPM.

The resistance of gas sensing element is the function of temperature and hence temperature measurement provision is also made in the system design. LM 35, a degree Celsius temperature sensor is used to measure the chamber temperature at which sensor response is recorded. 


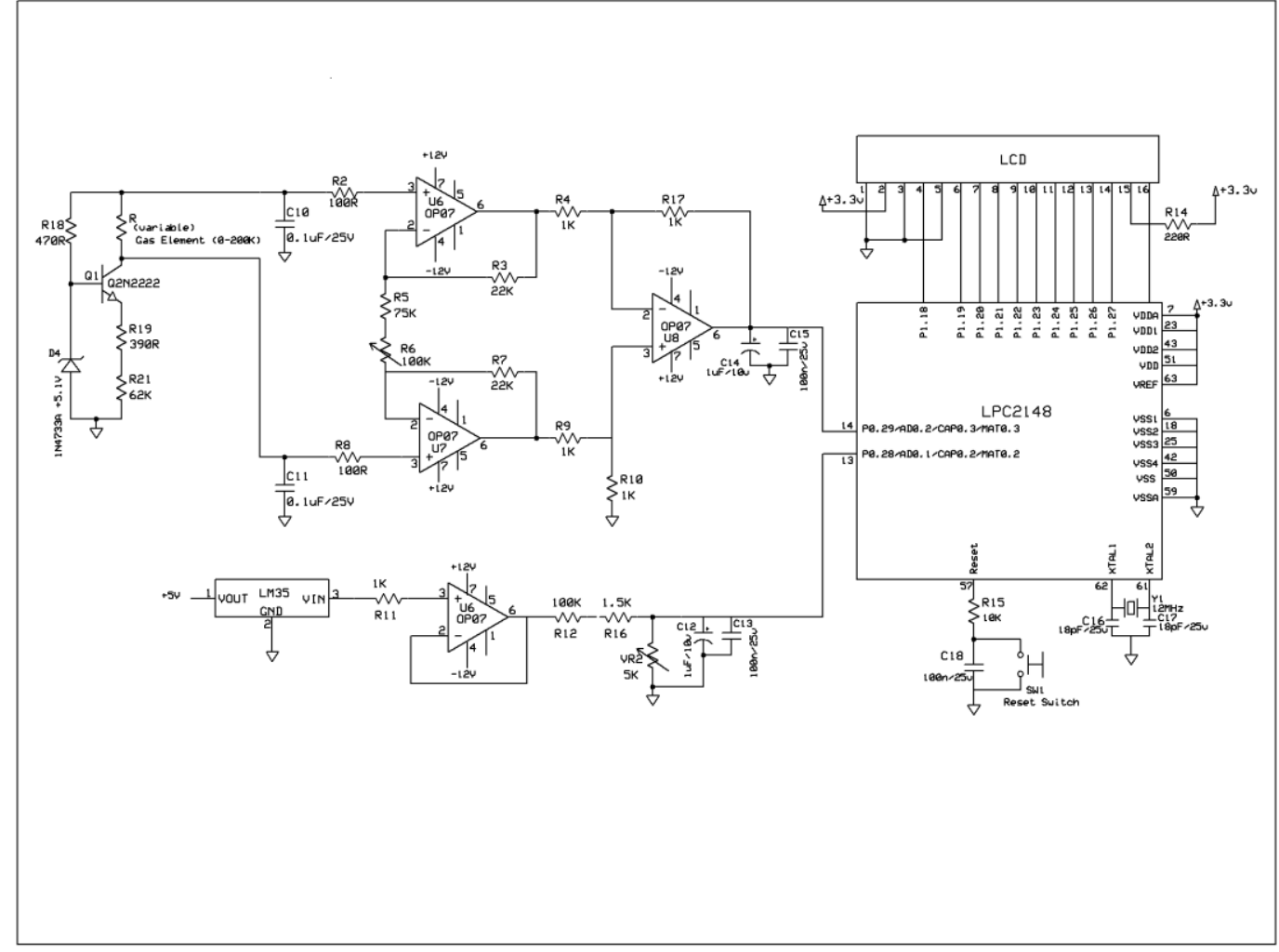

Figure 2 Circuit schematic of the gas sensing system

\section{B. System Implementation}

The sensing chamber made up of glass is mounted on a steel table. The sensing element in the form of pellet is placed in a sample holder and is projected from the bottom of the chamber through a cork. The whole set up is made airtight. Gas sensing chamber is provided with two valves, one for the gas inlet and the other as outlet. Using a flow meter and a regulator, oxygen is allowed to enter the sensing chamber at the rate of $20 \mathrm{cc}$ per minute. With variation in gas concentration, the resistance of sensing element also changes. The change in resistance of the sensing element causes the voltage change across the element as it is driven by the constant current source. This voltage drop is picked up by the instrumentation amplifier. Then the instrumentation amplifier output is fed to the analog channel of the ARM processor. Further the ARM7TDMI LPC2148 processor acquires the voltage through the analog channel, substitutes in the calibrated equation, computes it for the gas concentration in PPM, and then displays the same on 16X2 LCD display along with the temperature. Complete circuit schematic of the gas measurement system is shown in figure 2 and the software flowchart is shown in figure 3. 


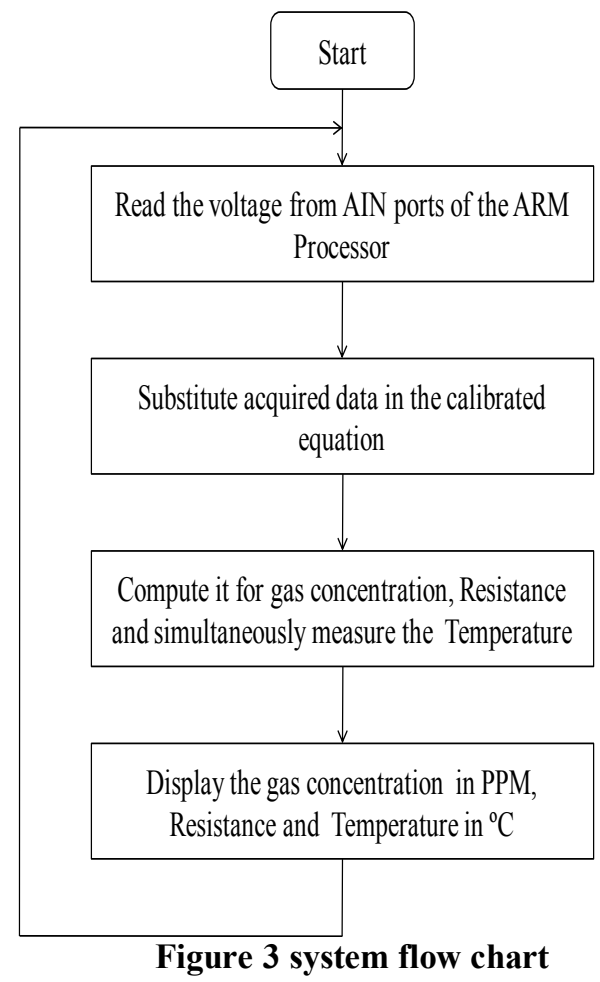

\section{Results And Discussion}

When the composites of different weight percentage of cerium oxide in polyaniline were tested for oxygen sensing, the variation of resistance was found to be maximum in the composite with $50 \mathrm{wt} \%$ of cerium oxide in polyaniline. Hence the designed system was tested for this sample. Initially the resistance of the sample for various known gas concentrations are recorded using high accuracy digital ohm meter. A plot of resistance $\mathrm{v} / \mathrm{s}$ known gas concentration in PPM is the reference for calibrating the designed gas sensing system. The performance of the calibrated gas sensing system is studied for oxygen sensing application. Figure 4 shows the plot of sensor resistance vs gas concentration in PPM obtained from the designed gas sensing system. ARM based gas sensing system measurements are found to be very close to the actual measurements made by the high accuracy digital multi meter. In case of metal oxide sensors the variation in resistance ranges from few kilo ohms to several mega ohms. As these sensors operate at high temperature heater design also must be made in the sensor system. Since the designed system operates at room temperature, heater design is eliminated.

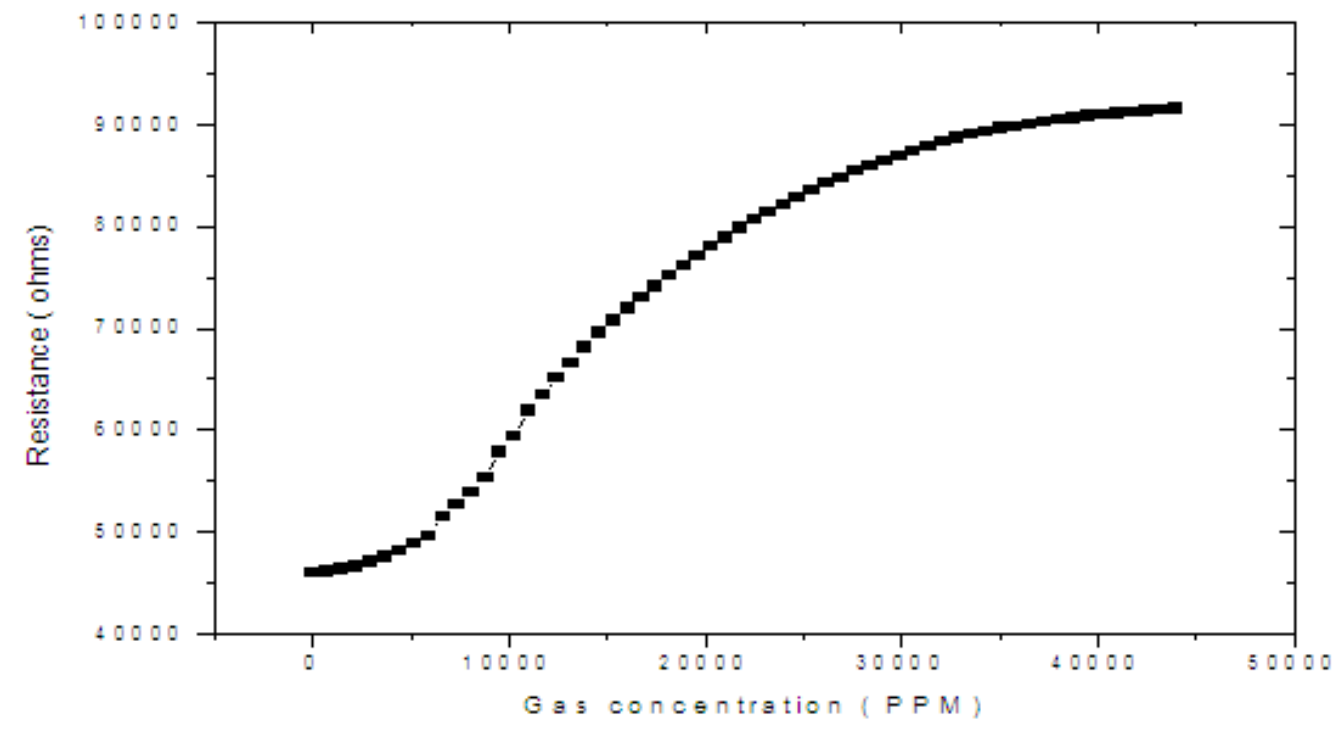




\section{Conclusion}

Design and development of a low cost gas sensing system is presented. Polyaniline/ Cerium oxide composite was synthesized by insitu polymerization method and pressed in the form of pellets. These pellets are used as oxygen sensing elements. Composite with 50 weight percent of cerium oxide in polyaniline was taken as sensing element since its resistance variation was maximum on exposure to oxygen gas. The sensing element is interfaced with analog front end signal conditioning circuit. ARM processor is used for data logging, computing the gas concentration in PPM and displaying the results. The sensor readout circuit is based on resistance to voltage conversion technique. The designed system measures the gas concentration with an accuracy of less than $\pm 1 \%$ and exhibits a linear response up to $200 \mathrm{~K} \Omega$. Provision can also be made to measure higher resistances by employing attenuators at the input of the ARM processor.

\section{References}

[1]. B.P.J. Lacy Costello, R.J. Ewen, N. Guernion, N.M. Ratcliffe, Highly sensitive mixed oxide sensors for the detection of ethanol, Sens. Actuators B Chem.87,2002, 207-210

[2]. S.Chi Tsang,C.Bulpitt, Rare eatrh oxide sensors for ethanol analysis, Sens.Actuators B Chem. 52,1998, 226-235

[3]. C.Garzella, E.Comini, E.Bontempi, L.E.Depero. C.Frigeri, G.Sberveglieri, Sol-gel $\mathrm{TiO}_{2}$ and W/TiO ${ }_{2}$ nanostructured thin films for control of drunken driving, Sens.Actuators B Chem. 83,2002, 230-237

[4]. M Ivanovskaya, P.Bogdanov, G.Faglia,P.Nelli,G.Sberveglieri, A.Taroni, On the role of catalytic additives in gas-sensitivity of SnO2-Mo based thin film sensors, Sens. Actuators B Chem. 77, 2001, 268-274

[5]. M. Mabrook, P. Hawkins, A rapidly- responding sensor for benzene, methanol and ethanol vapoursbased on films of titanium dioxide dispersed in a polymer operating at room temperature, Sens. Actuators B Chem. 75, 2001,197-202

[6]. Jinkawa T, Sakai G, Tamaki J, Miura N, Yamazoe N, Relationship between ethanol gas sensitivity and surface catalytic properties of tin oxide sensors modified with acidic or basic oxides, J. Mol. Catal. A. 155 , 2000, 193-200

[7]. J. Sarfraz, P. Ihalainen, A. Maattanen, J. Peltonen, M. Linden, Printed hydrogen sulfide gas sensor on paper substrate based on polyaniline composite, Thin Solid Films, 534,2013, 621-628

[8]. Sujata Pramanik, Gautam Das and Niranjan Karak, Facile preparation of polyaniline nanofibers modified bentonite nanohybrid for gas sensor application, RSC Adv.3,2013, 4574-4581

[9]. Chuanjun Liu,Kenshi Hayashi,Kiyoshi Toko, Au nanoparticles decorated polyaniline nanofiber sensor for detecting volatile sulfur compounds in expired breath, Sens. Actuators B:Chem,.161,2012, 504-509

[10]. Iole Venditti, Ilaria Fratoddi, Maria Vittoria Russo and Andrea Bearzotti, A nanostructured composite based on polyaniline and gold nanoparticles: synthesis and gas sensing properties, Nanotechnology 24, 2013,155503

[11]. Aashis S. Roy, Ameena Parveen, M V N Ambika Prasad, Koppalkar R. Anilkumar, Influence of $\mathrm{Ta}_{2} \mathrm{O}_{5}$ on polyaniline surface for liquid petroleum gas sensing applications, Sensor Review, 32 ,2012, 163 - 169

[12]. J.Wang, S.P.Chen, M.Shan Lin, Use of different electro polymerazation conditions for controlling the size exclusion selectivity at PANI,PPY and polyphenol films, J.Electroanal.Chem.273,1989, 231

[13]. G..R.Ruschau, R.E.Newnham, J.Runt, B.E.Smith, 3D ceramic/polymer composite chemical sensors , Sens.Actuators B 20 ,1989, 269-275

[14]. Hua Bai and Gaoquan Shi, Gas sensors based on conducting polymers, Sensors 2007,7, 267-307

[15]. Sarswati Koul, R Chandra, S.K.Dhawan, Conducting polyaniline composite: a reusable sensor material for aqueous ammonia , Sens. actuators $75,2001,151-159$

[16]. R. M. Chaudhari, V. B. Gaikwad, P. D. Hire, R. L. Patil,S. D. Shinde, N. U. Patil, G. H. Jain, Studies of Gas Sensing Performance of Barium Zirconate $\left(\mathrm{BaZrO}_{3}\right)$, Sens.and Transducers, 127, 2011,76-87

[17]. S.Hossein Hosseini, S.Hossein Abdi Oskooei, Ali Akbar Entezami, Toxic gas and vapour detection by polyaniline gas sensors, Iranian polymer Jornal,14(4),2005, 333-344

[18]. Noria Izu,Woosuck Shin,Norimitsu Murayama, Shuzo Kanzaki, Resistive oxygen gas sensors based on $\mathrm{CeO}_{2}$ fine powder prepared using mist pyrolysis, Sens. Actuators B, 87,2007,95-98

[19]. R.Bene, I.V.Perczel, F.Reti, F.A.Meyer, M.Fleisher, H.Meixner, Chemical reactions of acetone and $\mathrm{NO}^{-}$by a CeO $\mathrm{C}_{2}$ thin film, Sens. Actuators B ,71,2000, 36-41.

[20]. Liao,H.X.Mai,Q.Yuan,H.B.Lu,J.C.Li,C.Liu,C.H.Yan,Z.X.Shen and T.Yu, Single $\mathrm{CeO}_{2}$ nanowire gas sensor supported with Pt nanocrystals: Gas sensitivity, surface bond states and chemical mechanism, J.Phys.Chem.C ,112,2008, 9061-9065.

[21]. N.Barsan and $U$ weimar, Understanding the fundamental principles of metal oxide based gas sensors ; the example of CO sensing with $\mathrm{SnO}_{2}$ sensors in the presence of humidity , J.Phys.: Condens.Matter ,15,2003,R813-R839

[22]. H.V.Shrumer and J.W.Gardner, Odor discrimination with an electronic nose, Sens.Actuators B: Chem., 8, 1992, 1-11

[23]. J.W.Gardner, M.Vidic, P.Ingleby, A.C.Pike,J.E.Brignell, P.Scivier,P.N.Bartlett,A.J.Duke,and J.M.Elliott, Response of a poly(pyrrole) resistive micro-bridge to ethanol vapor, Sens.Actuators B: Chem.,48, 1998,.289-295

[24]. D.Barrettino, M.Graf, M.Zimmermann, A.Hierlemann, H.Baltes, S.Hahn, N.Barsan, and U.Weimar, A smart single chip micro-hotplate-based chemical sensor system in CMOS-Technology, in Proc Int.Symp.Circuits and Systems, 2002, 157-160 